\title{
Intradermal immunization with combined baculovirus and tumor cell lysate induces effective antitumor immunity in mice
}

\author{
MAMORU KAWAHARA ${ }^{1,2}$ and HIROSHI TAKAKU ${ }^{2,3}$ \\ ${ }^{1}$ Research and Development Department, Japan BCG Laboratory, Kiyose, Tokyo 204-0022; \\ ${ }^{2}$ Department of Life and Environmental Sciences and ${ }^{3}$ High Technology Research Center, \\ Chiba Institute of Technology, Narashino, Chiba 275-0016, Japan
}

Received August 1, 2013; Accepted September 13, 2013

DOI: $10.3892 /$ ijo.2013.2125

\begin{abstract}
Although tumor lysate contains all the potential helper and killer epitopes capable of stimulating $\mathrm{T}$ cells, it is difficult to use as a cancer vaccine because it suppresses dendritic cell (DC) function. We report that wild-type baculovirus possesses an adjuvant effect to improve the immunogenicity of tumor lysate. When mice were administered CT26 tumor cell lysate combined with baculovirus intradermally, antitumor immunity was induced and rejection of CT26 tumor growth was observed in $40 \%$ of the immunized mice. In contrast, such antitumor immunity was not elicited in mice inoculated with tumor cell lysate or baculovirus alone. In tumor-bearing mice, which had previously received the combined baculovirus and tumor lysate vaccine, the established tumors were completely eradicated by administering a booster dose of the combined vaccine. This antitumor effect was attributed to tumor-specific $\mathrm{T}$ cell immunity mediated primarily by $\mathrm{CD}^{+} \mathrm{T}$ cells. Baculovirus also strongly activated DCs loaded with tumor lysate. Increased interleukin (IL)-6 and IL-12p70 production were also observed in DCs co-cultured with tumor cell lysate and baculovirus. Our study demonstrates that combined baculovirus and tumor lysate vaccine can effectively stimulate DCs to induce acquired antitumor immunity.
\end{abstract}

\section{Introduction}

There is currently no effective therapy against recurrent tumors, which may occur after surgical treatment of the primary tumor. Thus, suppression of tumor recurrence in cancer patients is important. It was recently reported that gastric cancer patients receiving postoperative adjuvant chemotherapy showed a $5.8 \%$ increase in their 5-year overall survival compared to those

Correspondence to: Dr Mamoru Kawahara, Research and Development Department, Japan BCG Laboratory, 3-1-5 Matsuyama, Kiyose, Tokyo 204-0022, Japan

E-mail: kawahara@bcg.gr.jp

Key words: baculovirus, adjuvant, CT26, immunotherapy, vaccine who received surgery alone (1). This indicates the significance of adjuvant chemotherapy and also suggests the necessity for a more powerful adjuvant therapy to resist tumor recurrence.

$\mathrm{CD}^{+}$cytotoxic T lymphocytes (CTLs) are a major component of antitumor immune responses and CTL responses have been shown to be effective in eliminating tumors in animal models (2). Thus, tumor immunotherapy that induces tumorreactive CTLs is a good approach to investigate because CTLs are expected to disperse throughout the whole body in a cancer patient and destroy tumor cells if the tumor cells exist in the patient. Short peptides and proteins recognized by $\mathrm{CD}^{+}$ $\mathrm{T}$ cells have been used as cancer vaccines (3); however, alone, these antigens elicit weak antitumor immune responses in vivo and the efficacy of the therapies has shown only modest benefit so far in clinical studies (4). Previous studies have also shown that vaccination with peptides recognized by $\mathrm{CD}^{+} \mathrm{T}$ cells caused tolerance against tumors, so that the tumor growth was enhanced (5-7). These studies suggest the limitation of traditional approaches for stimulating $\mathrm{CD}^{+} \mathrm{T}$ cells with peptides containing only killer epitopes.

Several studies have shown that $\mathrm{CD}^{+}{ }^{+} \mathrm{T}$ cells also play a critical role in the development of a therapeutic antitumor immune response (8-10). In addition, $\mathrm{CD}^{+} \mathrm{T}$ cells are essential for generating $\mathrm{CD}^{+} \mathrm{T}$ memory cells $(11,12)$. Therefore, autologous tumor tissue surgically removed from a patient should be suitable to use as a source of cancer vaccine for suppressing tumor recurrence in the patient, because, unlike peptide-based vaccines, tumor cells should contain all potential MHC class I and MHC class II epitopes capable of stimulating $\mathrm{CD}^{+}$and $\mathrm{CD}^{+} \mathrm{T}$ cells, respectively. However, in some experiments, when tumor lysates were employed as vaccines, dendritic cells (DCs) often failed to function efficiently in tumor-bearing hosts due to pronounced immune suppression (13-15). Tumor cells are also known to alter expression of DC cell-surface molecules (e.g., MHC class II, CD116), decrease migration to draining lymph nodes and suppress cytokine release (16-18). Therefore, development of a new effective adjuvant that will improve the effectiveness of a cancer vaccine based on tumor lysates and stimulate Th1-type antitumor immunity represents a potential new therapeutic tool to prevent tumor recurrence.

Baculovirus Autographa californica multiple nuclear polyhedrosis virus, which has a 130-kb double-stranded circular 
DNA genome, is pathogenic for insects of Lepidoptera. Baculovirus has been widely used as a biopesticide $(19,20)$ and as a tool in recombinant protein-expression systems $(21,22)$. The virus can infect a range of mammalian cell types, but it does not replicate in the cells; this property leads to recombinant baculoviruses with a mammalian expression promoter that can be used as a tool for gene therapy (23-27). It was recently shown that baculovirus strongly stimulated the production of cytokines such as type I interferon, tumor necrosis factor- $\alpha$ and interleukin (IL)-1 in mammalian cells $(28,29)$. It was also demonstrated that intravenous injection of mice with baculovirus markedly activated natural killer (NK) cells, resulting in the induction of antitumor immunity (30). In addition, intratumoral inoculation with DCs that were stimulated in vitro with baculovirus suppressed tumor growth in mouse models $(31,32)$. These reports indicate that baculovirus efficiently activates innate immunity in mice. The next step in the study of the baculovirus adjuvant effect is to investigate whether or not baculovirus-stimulated innate immunity can lead to the induction of antigen-specific acquired immunity.

In the present study, we focus on the feasibility of baculovirus as an adjuvant for cancer immunotherapy. We prepared a tumor vaccine consisting of baculovirus and tumor cell lysate and examined whether or not intradermal (i.d.) inoculation with the combined baculovirus and tumor cell lysate vaccine effectively induces tumor-specific acquired immunity in a mouse model.

\section{Materials and methods}

Mice and cell lines. Four-week-old female BALB/c mice (Nippon SLC, Shizuoka, Japan) were used in a P2 level animal facility at Chiba Institute of Technology, Chiba, Japan. The study was conducted in the experimental animal area under the guidance of an institutional committee for biosafety and animal experiments. Spodoptera frugiperda (Sf-9) cells were cultured at $28^{\circ} \mathrm{C}$ in Sf-900 II medium (Invitrogen, Carlsbad, CA, USA). CT26 murine colon carcinoma cell line was purchased from American Type Culture Collection (ATCC; Manassas, VA, USA) and maintained in RPMI-1640 (Wako Pure Chemical Industries, Osaka, Japan) supplemented with $10 \%$ fetal calf serum (Invitrogen), $100 \mathrm{U} / \mathrm{ml}$ penicillin and $100 \mu \mathrm{g} / \mathrm{ml}$ streptomycin (Sigma-Aldrich, St. Louis, MO, USA).

Purification of wild-type baculovirus. Wild-type baculovirus was purchased from BD Biosciences (San Jose, CA, USA) and propagated in Sf-9 cells. Baculovirus was purified as previously described (31) and the virus titer was determined using a plaque assay.

Preparation of vaccines. To prepare tumor cell lysate, CT26 cells were divided into $1.5 \mathrm{ml}$ tubes $\left(1 \times 10^{6} / 50 \mu \mathrm{l} \mathrm{PBS} / \mathrm{tube}\right)$ and the tubes were treated with 5 freeze-thaw cycles using liquid nitrogen and a $37^{\circ} \mathrm{C}$ water bath. The lysates were stored at $-80^{\circ} \mathrm{C}$ until use. To prepare combined baculovirus and tumor cell lysate vaccine, baculovirus $\left[1 \times 10^{8}\right.$ plaque forming units (pfu)] was added into the 1.5-ml tube described above and stored on ice until administration. One tube was used per individual mouse $\left(1 \times 10^{6}\right.$ cell lysate plus $1 \times 10^{8}$ pfu baculovirus/ mouse). As controls, CT26 cell lysate alone or baculovirus alone was also prepared.

Immunization with vaccines. CT26 cell lysate alone, baculovirus alone, or combined CT26 cell lysate and baculovirus were inoculated intradermally into the upper right flank of mice. Each immunization was performed once per week for three consecutive weeks. Seven days after the final vaccination, the CT26 cells $\left(5 \times 10^{4}\right)$ were transplanted subcutaneously in the lower right flank of the immunized mice. Tumor volume was measured using a slide caliper according to the following formula: tumor volume $\left(\mathrm{mm}^{3}\right)=$ length $\mathrm{x}$ (width) ${ }^{2} / 2$. Mice were monitored twice weekly for tumor growth and survival.

In vitro cytotoxicity assay. As effector cells, splenocytes were isolated from mice immunized with PBS, tumor cell lysate alone, baculovirus alone, or the combined baculovirus and tumor cell lysate 7 days after the final vaccination. CT26 cells $\left(1 \times 10^{4}\right)$ were used as target cells. The effector cells were co-cultured with the target cells at ratios of 50:1, 25:1 and 12.5:1 for $8 \mathrm{~h}$. The cytolytic activity was assessed using the CytoTox 96 Non-Radioactive Cytotoxicity Assay kit (Promega, Madison, WI, USA), according to the manufacturer's instructions.

FITC-dextran or tumor cell lysate uptake by DCs. To prepare murine DCs, bone marrow cells were harvested from the tibiae and femurs of BALB/c mice and depleted of red blood cells using red blood cell lysis buffer (Sigma-Aldrich). Bone marrow cells were cultured in RPMI-1640 medium containing $10 \% \mathrm{FCS}, 100 \mathrm{U} / \mathrm{ml}$ penicillin, $100 \mu \mathrm{g} / \mathrm{ml}$ streptomycin and $50 \mu \mathrm{M}$ 2-mercaptoethanol (Invitrogen), supplemented with $20 \mathrm{ng} / \mathrm{ml}$ of murine granulocyte-macrophage colony-stimulating factor (GM-CSF) (Miltenyi Biotech, Bergisch Gladbach, Germany). On days 3 and 6, the culture medium was replaced with fresh medium supplemented with GM-CSF. On day 8, non-adherent cells were collected and positively selected with anti-mouse CD11c microbeads (Miltenyi Biotech).

To assess how baculovirus affects the ability of DCs to capture an antigen, DCs $\left(5 \times 10^{5}\right)$ were suspended in culture medium and FITC-dextran (MW=40,000) (Sigma-Aldrich) was added at a final concentration of $1 \mathrm{mg} / \mathrm{ml}$, with or without baculovirus at a multiplicity of infection (MOI) of 50. The cells were incubated at $37^{\circ} \mathrm{C}$ for $2 \mathrm{~h}$. Stimulation with lipopolysaccharide (LPS, $1 \mu \mathrm{g} / \mathrm{ml}$ ) was employed as a positive control and the incorporation of FITC-dextran at $4^{\circ} \mathrm{C}$ was used as a negative control (non-specific binding). Cells were washed 3 times with cold PBS containing $2 \%$ FCS and blocked followed by labeled with anti-mouse CD16/32 and PE-conjugated antimouse CD11c monoclonal antibodies (mAbs), respectively (eBioScience, San Diego, CA, USA).

To analyze how baculovirus affects DC activation, DCs $\left(5 \times 10^{5}\right)$ were co-cultured with CT26 tumor cell lysate $\left(1 \times 10^{6}\right)$ in the presence or absence of the virus $(\mathrm{MOI}=50)$. Stimulation with LPS $(1 \mu \mathrm{g} / \mathrm{ml})$ was used as a positive control. The cells were incubated at $37^{\circ} \mathrm{C}$ for 48 or $96 \mathrm{~h}$ and then labeled with PE-conjugated anti-mouse CD11c and FITC-conjugated CD86 mAbs (eBioScience). Cells were analyzed using FACSCalibur with the CellQuest software (BD Bioscience). 
A
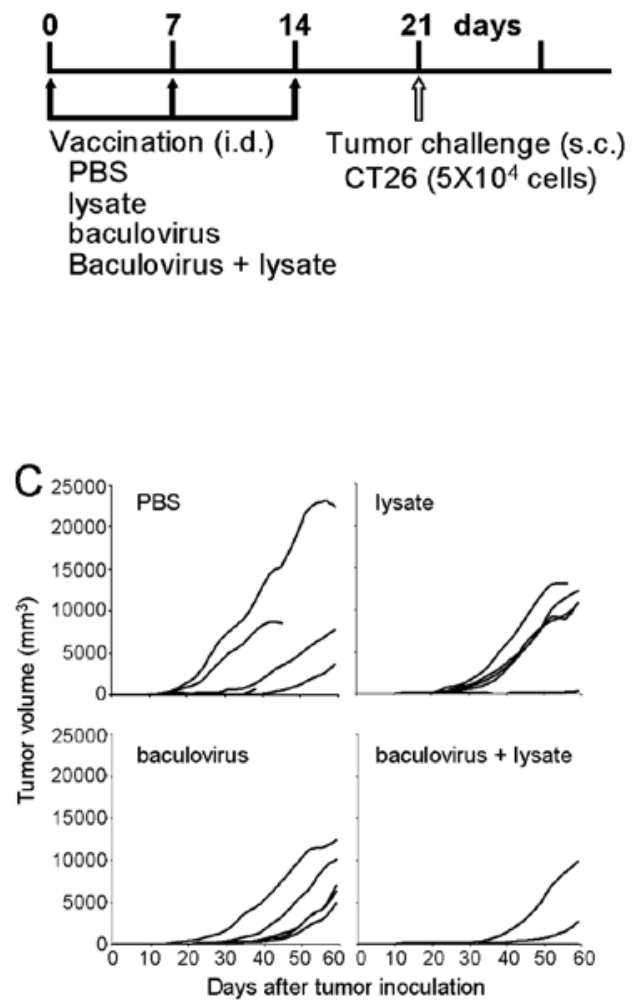

B

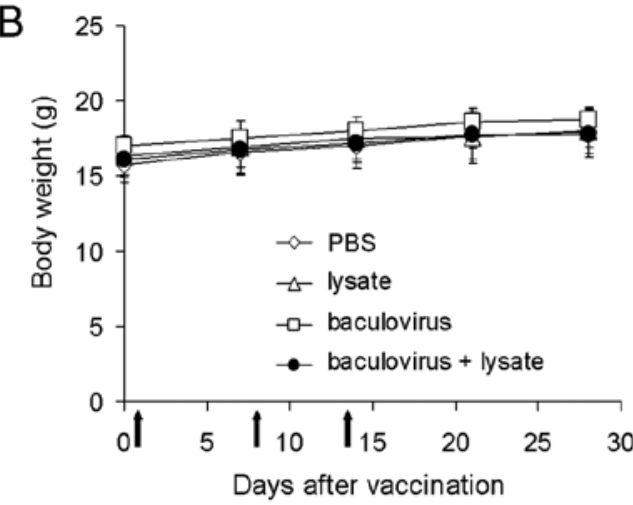

D

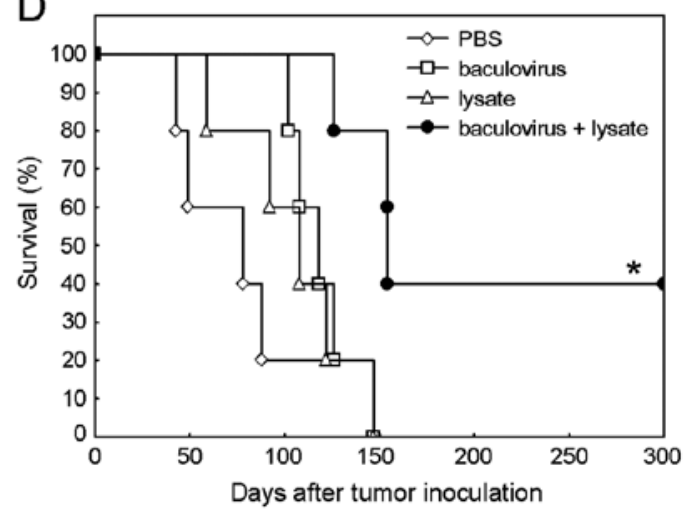

Figure 1. Vaccination with combined baculovirus and tumor cell lysate induces antitumor resistance. (A) Mice were immunized intradermally with PBS, tumor cell lysate alone, baculovirus alone, or the combined baculovirus and tumor cell lysate once per week for three consecutive weeks (5 mice per group). One week after the final vaccination, CT26 cells $\left(5 \times 10^{4}\right)$ were transplanted subcutaneously. (B) Body weight of the immunized mice was monitored to assess whether i.d. inoculation with baculovirus affects the growth of mice. (C) Tumor size was measured. Each line represents an individual mouse. (D) Survival was recorded. ${ }^{*} \mathrm{P}<0.05$, the group that received the combined vaccine compared to the groups that received PBS, tumor cell lysate alone, or baculovirus alone. Similar results were obtained in two independent experiments.

ELISA. DCs $\left(5 \times 10^{5}\right)$ were co-cultured with CT26 cell lysate $\left(1 \times 10^{6}\right)$ in the presence or absence of baculovirus (MOI $\left.=50\right)$ or LPS $(1 \mu \mathrm{g} / \mathrm{ml})$ for $48 \mathrm{~h}$ and then the culture supernatants were harvested. The production levels of mouse IL-6, IL-10 and IL-12p70 were measured using ELISA kits (eBioScience).

Statistical analysis. Statistical analyses were performed using a one-way analysis of variance (ANOVA) followed by the Tukey test for pair-wise comparisons; calculations were performed using the Statistica program (StatSoft, Tulsa, OK, USA). Survival data were plotted using the method of Kaplan-Meier and were analyzed using the log-rank test. Data are expressed as the mean \pm standard deviation (SD) and P-values $<0.05$ were considered significant.

\section{Results}

Induction of antitumor immunity by intradermal immunization with combined baculovirus and tumor cell lysate vaccine. To assess whether i.d. inoculation with baculovirus affects the growth of mice, we monitored the body weight of mice that received the combined baculovirus and tumor cell lysate vaccine once per week for three consecutive weeks (Fig. 1A). The body weight was also measured in control mice that received PBS, tumor cell lysate alone, or baculovirus alone (Fig. 1A). There was no difference in the increase of body weight between the four groups of mice (Fig. 1B), which suggests that the growth was not affected by i.d. baculovirus injection. However, induration was observed at the vaccination sites in mice receiving the combined baculovirus and tumor cell lysate, but not in the animals receiving baculovirus alone or tumor cell lysate alone.

To verify whether the combined baculovirus and tumor cell lysate vaccine can induce effective antitumor immunity, the combined vaccine, tumor cell lysate alone, or baculovirus alone was administered intradermally into the upper right flank of mice once per week for three consecutive weeks. At 1 week after the final vaccination, CT26 cells $\left(5 \times 10^{4}\right)$ were inoculated subcutaneously into the lower right flank of the mice (Fig. 1A) and $40 \%$ of the combined vaccine-immunized mice did not demonstrate tumorigenesis (Fig. 1C). However, tumorigenesis was detected in all of the mice inoculated with PBS, tumor cell lysate alone, or baculovirus alone during the observation period (Fig. 1C). Tumors were observable at 7 days after the tumor inoculation in all of the animals that received PBS, tumor cell lysate alone and baculovirus alone and in $60 \%$ of mice that received the combined vaccine. Although variance in the tumor growth was observed among animals in each group, the tumor growth was slower in mice immunized with the combined vaccine compared to mice that received 

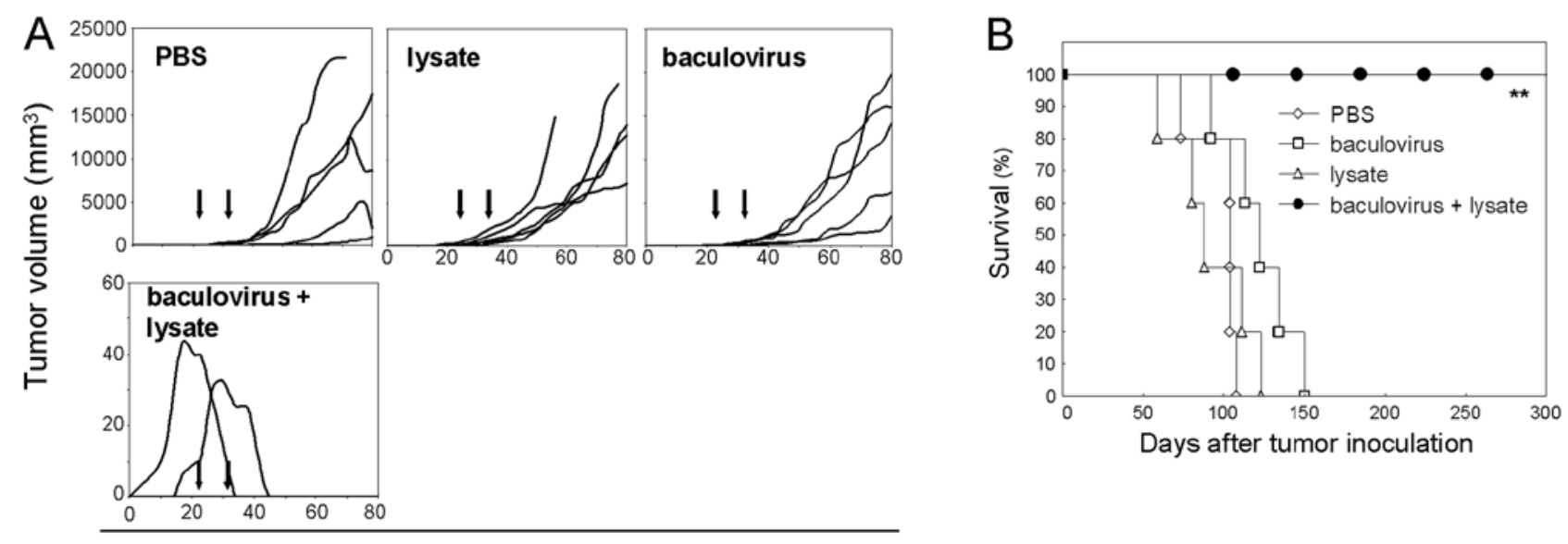

Days after tumor challenge

Figure 2. I.d. booster vaccination with combined baculovirus and tumor cell lysate eradicates the tumor. (A) Mice were vaccinated intradermally with PBS, tumor cell lysate alone, baculovirus alone, or the combined vaccine at weeks 0,1 and 3 and then CT26 cells (5x10 $)$ were inoculated subcutaneously at week 4 (5 mice per group). The booster vaccination with each vaccine was performed at days 21 and 31 after the tumor inoculation (arrows) and tumor size was measured. Each line represents an individual mouse. (B) Survival was monitored. ${ }^{* *} \mathrm{P}<0.01$, the group that received the combined vaccine compared to the groups that received PBS, tumor cell lysate alone, or baculovirus alone. Similar results were obtained in two independent experiments.

PBS, tumor cell lysate alone, or baculovirus alone (Fig. 1C). The median values of tumor size at day 38 after the tumor inoculation were $128.4,1322.8,2514.3$ and $463.9 \mathrm{~mm}^{3}$ for the groups receiving the combined vaccine, PBS, tumor cell lysate alone and baculovirus alone, respectively. Forty percent of the mice immunized with the combined vaccine did not demonstrate tumorigenesis during a 1-year follow-up period and the survival rate was significantly higher in mice immunized with the combined vaccine compared to that of mice injected with PBS, tumor cell lysate alone or baculovirus alone $(\mathrm{P}=0.02$, Fig. 1D).

Therapeutic effect of combined baculovirus and tumor cell lysate vaccine on established tumors. We next investigated whether the combined vaccine can effectively eradicate established tumors. Mice were vaccinated intradermally with PBS, tumor cell lysate alone, baculovirus alone, or the combined vaccines at days 0,7 and 14 , and then CT26 cells $\left(5 \times 10^{4}\right)$ were inoculated subcutaneously at day 21 (Fig. 1A). There was a marked difference in stimulation of the antitumor immunity among the four vaccination groups (Fig. 2A). Three out of five mice that received baculovirus plus tumor cell lysate became resistant to a challenge with the tumor (Fig. 2A). Because the remaining mice showed tumor growth, a therapeutic booster inoculation with baculovirus plus tumor cell lysate was administered at 21 and 31 days after the tumor inoculation. The booster vaccine was intradermally inoculated at a 1-2 mm distance around the tumor. Upon the first booster vaccination, one mouse showed a rapid regression of the tumor and the tumor volume of another mouse was also reduced gradually following the second booster vaccination (Fig. 2A). Eventually, the tumors were completely eradicated in each of two mice that received a booster dose of the combined baculovirus and tumor cell lysate (Fig. 2A). In addition, $100 \%$ of the mice survived for more than one year without tumor recurrence in the group that received the combined vaccine and the survival rate was significantly higher in this group compared to that in groups receiving PBS, baculovirus alone, or tumor cell lysate alone ( $\mathrm{P}=0.003$, Fig. $2 \mathrm{~B})$. However, tumorigenesis was observed in all of the mice immunized either with baculovirus alone or tumor cell lysate alone and mouse survival was not significantly different compared to that seen in PBS-injected control mice (Fig. 2). The mice inoculated with baculovirus alone or tumor cell lysate alone also received the i.d. therapeutic booster immunization with baculovirus or tumor cell lysate at 21 and 31 days after the tumor inoculation; however, the tumor in these animals did not regress (Fig. 2A).

Induction of antitumor $T$ cell immunity by intradermal immunization with combined baculovirus and tumor cell lysate. To investigate whether or not $\mathrm{CD}^{+} \mathrm{T}$ cells induced by the i.d. vaccination with the combined baculovirus and tumor cell lysate function by lysing the tumor cells, we conducted an in vitro cytotoxicity assay. Cytolytic activity against CT26 cells was detected when spleen cells obtained from the mice receiving the combined vaccine were co-cultured with target CT26 cells at ratios of 50:1, 25:1 and 12.5:1 (Fig. 3). The magnitude of the cytolytic activity in the combined vaccine group was considerably higher than that in the other groups that received PBS, tumor cell lysate alone, or baculovirus alone. This result demonstrates that i.d. immunization with the combined baculovirus and tumor cell lysate effectively induced tumor-reactive CTLs.

Effects of baculovirus on the ability of DCs to capture an antigen and their activation. To assess whether baculovirus affects the ability of DCs to take up an antigen, DCs were co-cultured with FITC-dextran as an antigen in the presence or absence of the virus. FITC-dextran uptake by DCs co-cultured with baculovirus was almost equivalent to that without the virus or with LPS $(32.9 \pm 5.6,30.0 \pm 5.8$ and $28.6 \pm 4.3 \%$, respectively; Fig. 4).

In addition, we investigated DC activation when the cells were co-cultured with CT26 cell lysate in the presence 


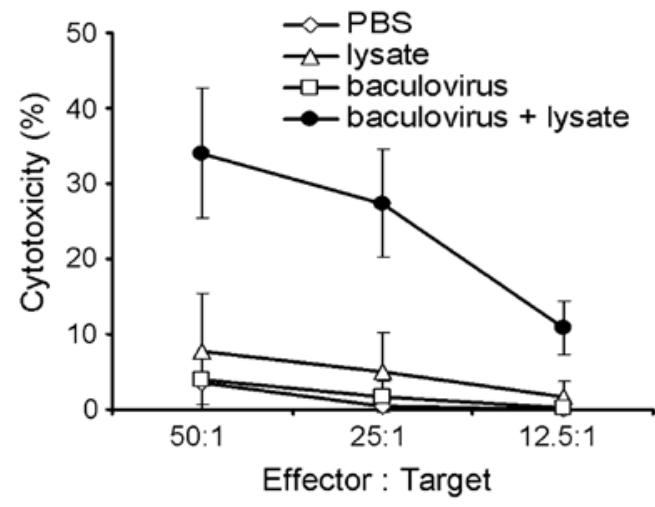

Figure 3. Vaccination with combined baculovirus and tumor cell lysate evokes cytolytic activity against CT26 cells. As effector cells, splenocytes were isolated from mice immunized with PBS, tumor cell lysate alone, baculovirus alone, or the combined baculovirus and tumor cell lysate 7 days after the final vaccination ( 5 mice per group). As target cells, CT26 cells $\left(1 \times 10^{4}\right)$ were used. The effector cells were co-cultured with the target cells at ratios of 50:1, 25:1 and 12.5:1 for $8 \mathrm{~h}$. Values are the mean \pm standard deviation of all animals per group. Similar results were obtained in two independent experiments.

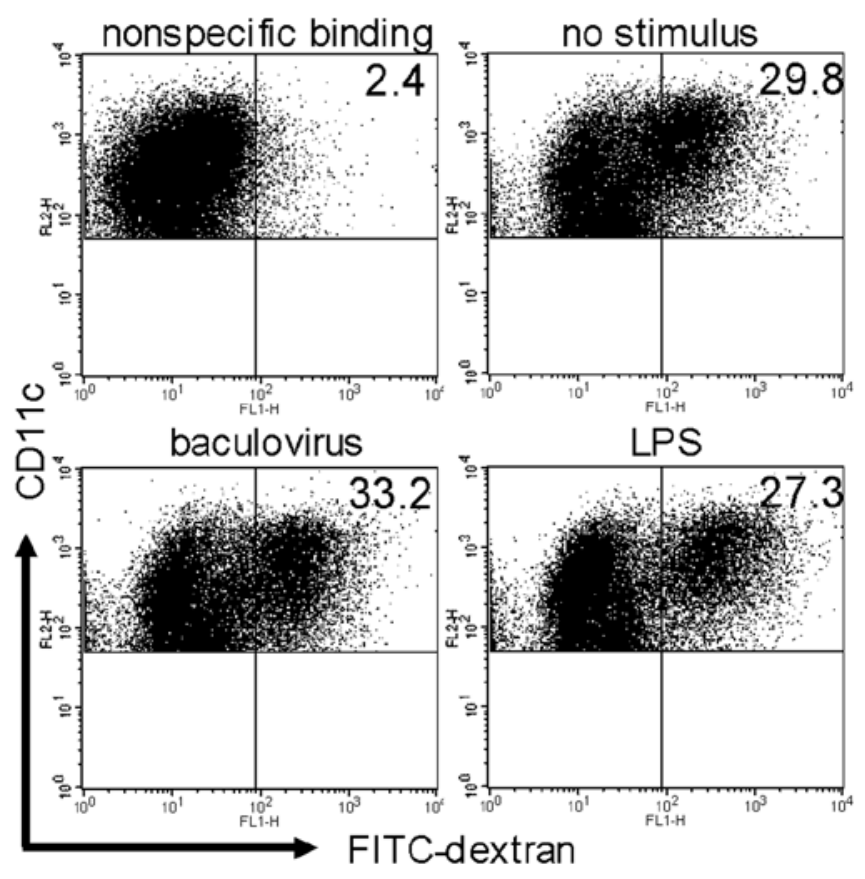

Figure 4. Comparison of FITC-dextran uptake by DCs in the presence or absence of baculovirus stimulation. DCs $\left(5 \times 10^{5}\right)$ were co-cultured with FITCdextran $(1 \mathrm{mg} / \mathrm{ml})$ in the presence or absence of baculovirus $(\mathrm{MOI}=50)$ at $37^{\circ} \mathrm{C}$ for $2 \mathrm{~h}$. DCs were co-cultured with FITC-dextran and LPS $(1 \mu \mathrm{g} / \mathrm{ml})$ as a positive control and the incorporation of FITC-dextran at $4^{\circ} \mathrm{C}$ was used as a negative control (non-specific binding). The frequency of uptake of FITC-dextran by DCs was measured by flow cytometry. Plots were gated on $\mathrm{CD} 11 \mathrm{c}^{+}$cells. Numbers in the upper right quadrant are the percentages of DCs taking up FITC-dextran. The representative data from one of the three experiments are shown.

or absence of the baculovirus. Following incubation with the lysate and virus for $48 \mathrm{~h}, 56.7 \pm 5.7 \%$ of DCs became CD86-positive, representing the activation of DCs, while the frequencies of CD86-positive DCs were $4.4 \pm 0.6 \%$ and $21.3 \pm 2.5 \%$ after incubation without the virus and with LPS, respectively ( $\mathrm{P}=0.0002$, Fig. $5 \mathrm{~A})$. Following incubation with the lysate and baculovirus for $96 \mathrm{~h}, 18.1 \pm 1.8 \%$ of DCs were CD86-positive, while the frequencies of CD86-positive DCs were $2.3 \pm 0.5 \%$ and $1.4 \pm 0.3 \%$ after incubation without the virus and with LPS, respectively ( $\mathrm{P}=0.0002$, Fig. 5B).

The production level of cytokines was also measured in the culture medium obtained from the above experiment (Fig. 5C). A high level of IL-6 production was observed in DCs co-cultured with baculovirus plus tumor lysate for $48 \mathrm{~h}$ compared to the level seen in DCs co-cultured with tumor lysate alone or with the lysate plus LPS. No IL-12p70 secretion was observed in DCs pulsed with tumor lysate alone; however, the cytokine production was elevated by adding baculovirus into DCs pulsed with tumor lysate. The level of IL-10 secretion from DCs co-cultured with tumor lysate plus baculovirus tended to be slightly higher, but not significantly different, compared to that from DCs co-cultured with tumor lysate alone; however, the production level from DCs co-cultured with baculovirus plus tumor lysate was lower than that from DCs co-cultured with tumor lysate plus LPS.

\section{Discussion}

Although baculovirus is widely used as both a tool to investigate gene transfer and a vaccine vector using a recombinant baculovirus (23-27), it remains unclear whether wild-type baculovirus possesses an adjuvant effect for a cancer vaccine when the virus is combined with a tumor antigen. We report here that i.d. inoculation with combined freeze-thaw tumor cell lysate and wild-type baculovirus effectively induces antitumor immune responses, mainly mediated by tumor-reactive $\mathrm{CD}^{+} \mathrm{T}$ cells, in a mouse model. Survival was significantly prolonged in mice vaccinated with the combined baculovirus and tumor cell lysate compared to that in animals inoculated either with baculovirus alone or tumor cell lysate alone. In addition, established tumors in mice that had previously been vaccinated with the combined baculovirus and tumor cell lysate were completely eradicated when the combined vaccine was administered around the tumors. These findings suggest that this vaccine strategy using wild-type baculovirus as an adjuvant is effective in preventing postoperative tumor recurrence and for treatment of recurrent tumors.

Many peptides containing killer epitopes were identified and are being used as cancer vaccines to induce tumor-reactive CTLs; however, clinical benefits of the vaccines are modest $(3,4)$. One reason for the modest effect of the vaccines may be because this vaccine strategy targets CTL stimulation only. Several studies have reported the critical role of $\mathrm{CD}^{+} \mathrm{T}$ cells in inducing antitumor responses (8-10) and in generating $\mathrm{CD}^{+}$ T memory cells $(11,12)$. These reports prompted us to consider tumor lysates as a useful cancer vaccine since tumor lysates should contain helper epitopes and killer epitopes. However, tumor lysate was reported to give rise to DC dysfunction (13-15) and moreover, it was also shown that DC was not able to enhance a cytotoxic activity even by stimulating with cell lysate (33).

In the present study, when tumor cells were transplanted subcutaneously into mice that had been intradermally vaccinated with the combined baculovirus and tumor cell lysate, baculovirus alone, or tumor cell lysate alone, the combination 
A

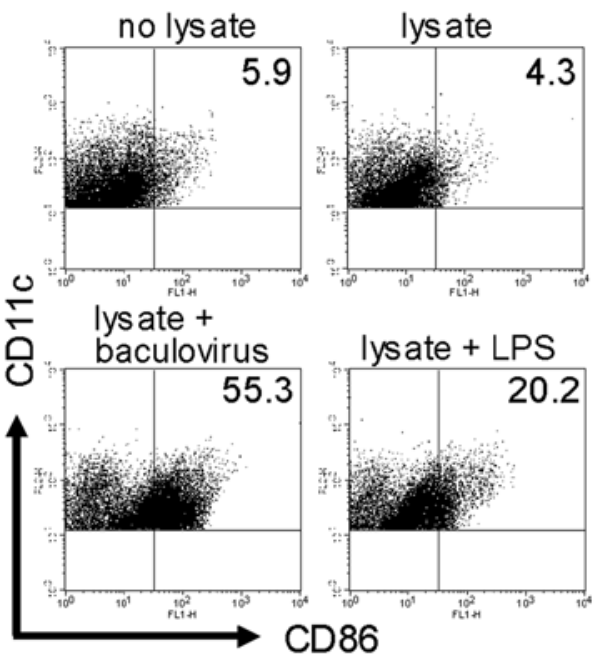

B

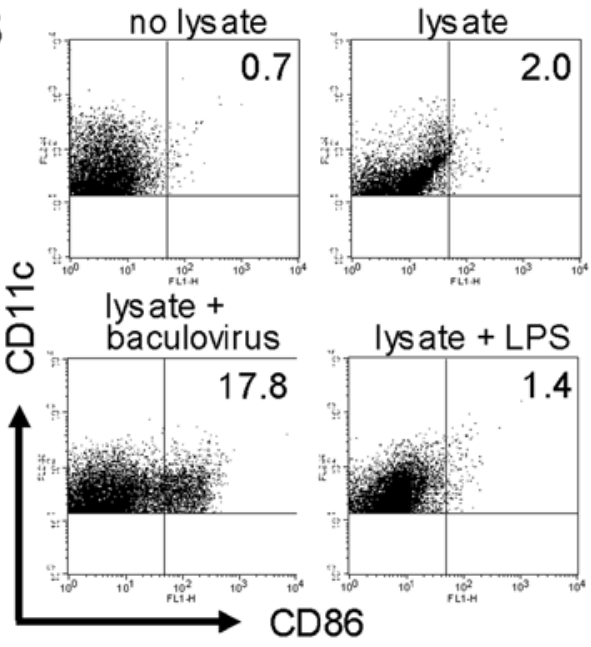

C
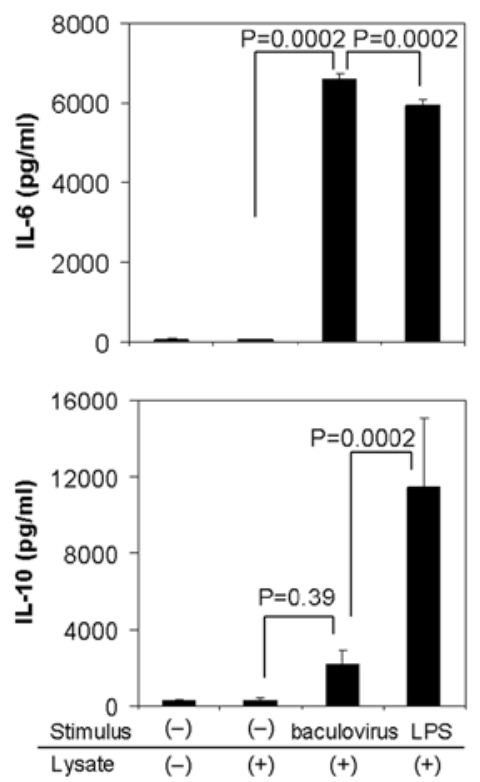

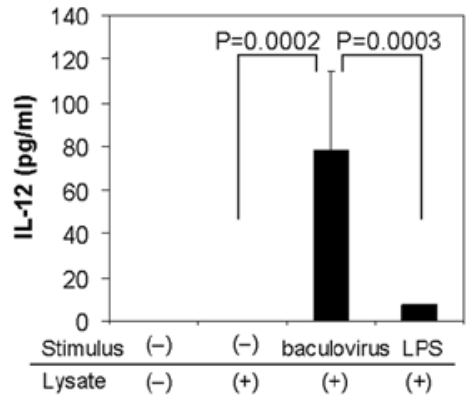

Figure 5. Comparison of activation of DCs co-cultured with CT26 cell lysate in the presence or absence of baculovirus stimulation. DCs $\left(5 \times 10^{5}\right)$ were cocultured with tumor cell lysate $\left(1 \times 10^{6}\right)$ in the presence or absence of baculovirus (MOI $\left.=50\right)$ at $37^{\circ} \mathrm{C}$ for $48 \mathrm{~h}(\mathrm{~A})$ or $96 \mathrm{~h}(\mathrm{~B})$. DCs were co-cultured with tumor cell lysate and LPS $(1 \mu \mathrm{g} / \mathrm{ml})$ as a positive control. The frequency of CD86 cells was measured by flow cytometry. All plots were gated on CD11 $\mathrm{c}^{+}$ cells. Numbers in the upper right quadrant are the percentages of CD86-positive DCs. The representative data from one of the five experiments are shown. (C) Levels of IL-6, IL-10 and IL-12p70 production were measured in culture medium from DCs co-cultured with tumor cell lysate in the presence or absence of the indicated stimulation for $48 \mathrm{~h}$. Similar results were obtained in two independent experiments.

vaccine was found to provide strong antitumor immunity that suppressed tumorigenesis compared to baculovirus alone or tumor cell lysate alone. This result indicates that baculovirus would not share the same peptide recognized by $\mathrm{T}$ cells as does the CT26 cell; baculovirus itself cannot induce antitumor immunity, but exerts a strong adjuvant effect to evoke antitumor immunity when the virus plus freeze-thawed tumor cell lysate is inoculated intradermally. We further demonstrated that such antitumor immunity induced by the i.d. immunization with the combined baculovirus and tumor cell lysate vaccine should be associated with $\mathrm{CD}^{+} \mathrm{T}$ cells using a cytotoxicity assay. A high level of cytolytic activity was detected in mice vaccinated with the combined baculovirus and tumor cell lysate vaccine compared to that in mice inoculated with baculovirus alone or tumor lysate alone. These results suggest the feasibility of using baculovirus as a vaccine adjuvant, which can help to strongly induce tumor-reactive CTLs.

When DCs were co-cultured with FITC-dextran in the presence or absence of baculovirus, the antigen uptake by DCs co-cultured with the virus was almost equivalent to that without the virus. However, when DCs were pulsed with tumor cell lysate for $48 \mathrm{~h}$, the frequency of CD86-positive mature DCs was substantially higher in the presence of baculovirus compared to that observed in the absence of the stimulus. The mature DCs remained viable $96 \mathrm{~h}$ following incubation with tumor cell lysate and the baculovirus. These results indicate that although baculovirus does not enhance the ability of DCs to take up an antigen, it strongly stimulates DCs loaded with tumor cell lysate. DCs are needed to prime naïve T cell responses so that they undergo the maturation process (34). 
We demonstrated here that baculovirus helps DCs stimulated with tumor lysate to become mature and also helps to maintain them.

We also found that cytokine production was enhanced by baculovirus-induced stimulation of tumor cell lysate-loaded DCs. It should be noted that a higher level of IL- 6 was produced by DCs co-cultured with tumor cell lysate plus the virus compared to DCs co-cultured with the tumor cell lysate alone. IL-6 has been shown to play an important role in T cell activation because of its ability to overcome the suppressive effect of regulatory $\mathrm{T}$ cells (Tregs) (35). Tregs have been reported to downregulate $\mathrm{T}$ cell responses (36-39) and Treg depletion has also been shown to contribute to enhanced antitumor immunity $(40,41)$. Accordingly, it is possible that high levels of IL-6 secreted by baculovirus-activated DCs might block suppression mediated by Tregs. In addition, IL-12, which is important to induce Th1-type immune responses, was secreted by DCs stimulated with tumor cell lysate plus baculovirus, whereas no IL-12 was produced by DCs co-cultured with tumor cell lysate alone. These results also indicate that combined baculovirus and tumor lysate vaccine can elicit effective DC activation and secretion of IL- 6 and IL-12, leading to the induction of antitumor immunity.

Why can the combined baculovirus and tumor cell lysate vaccine elicit tumor-reactive CTLs? In general, for the induction of antigen-specific CTLs, a killer epitope is required to be presented on MHC class I molecules of antigen-presenting cells. It has been reported that baculovirus infects mammalian cells through the cell-surface phospholipid (42). Thus, one possible explanation is that baculovirus might bind to freezethawed cells via a cell-surface phospholipid and the resulting complex consisting of the virus and lysate might be captured and endocytosed by DCs. The complex may then be released from the endosomal compartment to the cytosol by fusing with the viral envelope containing the tumor lysate into endosomal membrane and possibly lead to the cross-presentation of the tumor antigen to MHC class I molecules. In addition, these DCs effectively mature and become active by stimulation with baculovirus and IL-6 and IL-12 are also simultaneously secreted by the DCs, perhaps resulting in the induction of tumor-reactive CTLs.

As described in the present study, we observed that i.d. immunization with tumor cell lysate alone did not elicit effective antitumor immunity. DCs took up FITC-dextran in the absence of baculovirus because immature DCs possess the ability to take up an antigen. Therefore, DCs must have taken up tumor cell lysate in the absence of baculovirus; however, only a few DCs matured and these secreted little IL-6 and IL-12. These results may lead to the induction of tolerance against tumors, so that such an immune condition might increase the tumor growth acceleration in mice immunized with tumor cell lysate alone compared to that in mice injected with PBS (2514.3 and $1322.8 \mathrm{~mm}^{3}$, respectively). Because it is necessary to trigger innate immunity for subsequent effective acquired immunity (43), i.d. inoculation with tumor lysate alone may be insufficient to stimulate innate immunity, so that acquired antitumor immunity may not be fully stimulated.

In the present study, prophylactic vaccination with the combined baculovirus and tumor cell lysate suppressed tumor growth in 40-60\% of mice (Figs. 1 and 2). Although the remaining mice showed tumor growth, therapeutic booster vaccination with the combined vaccine completely eradicated the established tumors. The booster vaccination with the combined baculovirus and tumor cell lysate may effectively activate the antigen-captured DCs and these DCs may then stimulate tumor-specific memory $\mathrm{CD}^{+} \mathrm{T}$ cells through the cross-presentation of the tumor antigen. These DCs concomitantly secrete high level of IL-6 and IL-12, possibly resulting in the induction of effective antitumor responses.

In conclusion, we have demonstrated that baculovirus can effectively activate DCs pulsed with tumor lysate and that a strong antitumor immunity can be induced by i.d. immunization with combined baculovirus and tumor lysate. Baculovirus is not able to replicate in mammalian cells and the virus is also widely used as an agricultural insecticide, suggesting that baculovirus is safe for humans. Our findings reported here suggest the possibilities that baculovirus can make tumor lysate useful as a cancer vaccine and that the virus can be used as an effective adjuvant for cancer immunotherapy.

\section{Acknowledgements}

We would like to thank Drs Tomoyuki Suzuki (Chiba Institute of Technology), Noboru Hagiwara, Akira Hashimoto and Kazuhiro Matsuo (Japan BCG Laboratory) for their insightful comments during our discussion of this study.

\section{References}

1. Paoletti X, Burzykowski T, Michiels S, Ohashi Y, Pignon JP, Rougier P, Sakamoto J, Sargent D, Sasako M, Van Cutsem E and Buyse M: Benefit of adjuvant chemotherapy for resectable gastric cancer: a meta-analysis. JAMA 303: 1729-1737, 2010.

2. Ochsenbein AF: Principles of tumor immunosurveillance and implications for immunotherapy. Cancer Gene Ther 9: 1043-1055, 2002.

3. Kirkwood JM, Butterfield LH, Tarhini AA, Zarour H, Kalinski P and Ferrone S: Immunotherapy of cancer in 2012. CA Cancer J Clin 62: 309-335, 2012.

4. Rosenberg SA, Yang JC and Restifo NP: Cancer immunotherapy: moving beyond current vaccines. Nat Med 10: 909-915, 2004.

5. Toes RE, Blom RJ, Offringa R, Kast WM and Melief CJ: Enhanced tumor outgrowth after peptide vaccination. Functional deletion of tumor-specific CTL induced by peptide vaccination can lead to the inability to reject tumors. J Immunol 156: 3911-3918, 1996.

6. Toes RE, Offringa R, Blom RJ, Melief CJ and Kast WM: Peptide vaccination can lead to enhanced tumor growth through specific T-cell tolerance induction. Proc Natl Acad Sci USA 93: 7855-7860, 1996

7. Muraoka D, Kato T, Wang L, Maeda Y, Noguchi T, Harada N, Takeda K, Yagita H, Guillaume P, Luescher I, Old LJ, Shiku H and Nishikawa H: Peptide vaccine induces enhanced tumor growth associated with apoptosis induction in $\mathrm{CD} 8^{+} \mathrm{T}$ cells. J Immunol 185: 3768-3776, 2010.

8. Bour H, Horvath C, Lurquin C, Cerottini JC and MacDonald HR: Differential requirement for CD4 help in the development of an antigen-specific $\mathrm{CD}^{+} \mathrm{T}$ cell response depending on the route of immunization. J Immunol 160: 5522-5529, 1998.

9. Pardoll DM and Topalian SL: The role of $\mathrm{CD}^{+} \mathrm{T}$ cell responses in antitumor immunity. Curr Opin Immunol 10: 588-594, 1998.

10. Toes RE, Ossendorp F, Offringa R and Melief CJ: CD4 T cells and their role in antitumor immune responses. J Exp Med 189: 753-756, 1999.

11. Janssen EM, Lemmens EE, Wolfe T, Christen U, von Herrath MG and Schoenberger SP: CD4 ${ }^{+} \mathrm{T}$ cells are required for secondary expansion and memory in $\mathrm{CD}^{+} \mathrm{T}$ lymphocytes. Nature 421 : 852-856, 2003.

12. Shedlock DJ and Shen H: Requirement for CD4 T cell help in generating functional CD8 T cell memory. Science 300: 337-339, 2003. 
13. Lizée G, Rasvanyi LG, Overwijik WW and Hwu P: Immunosuppression in melanoma immunotherapy: potential opportunities for intervention. Clin Cancer Res 12: S2359-S2365, 2006.

14. Jackson AM, Mulcathy LA, Zhu XW, O'Donnell D and Patel PM: Tumor-mediated disruption of dendritic cell function: inhibiting the MEK1/2-p44/42 axis restores IL-12 production and Th1-generation. Int J Cancer 123: 623-632, 2008.

15. Yang DH, Park JS, Jin CJ, Kang HK, Nam JH, Rhee JH, Kim YK, Chung SY, Choi SJN, Kim HJ, Chung IJ and Lee JJ: The dysfunction and abnormal signaling pathway of dendritic cells loaded by tumor antigen can be overcome by neutralizing VEGF in multiple myeloma. Leuk Res 33: 665-670, 2009.

16. Schnurr M, Toy T, Stoitzner P, Cameron P, Shin A, Beecroft T, Davis ID, Cebon J and Maraskovsky E: ATP gradients inhibit the migratory capacity of specific human dendritic cell types: implications for P2Y11 receptor signaling. Blood 102: 613-620, 2003.

17. Pinzon-Charry A, Maxwell T and Lopez JA: Dendritic cell dysfunction in cancer: a mechanism for immunosuppression. Immunol Cell Biol 83: 451-461, 2005.

18. Bennaceur K, Popa I, Portoukalian J, Bethier-Vergnes O and Peguet-Navarro J: Melanoma-derived gangliosides impair migratory and antigen-presenting function of human epidermal Langerhans cells and induce their apoptosis. Int Immunol 18 : 879-886, 2006.

19. Stewart LM, Hirst M, López-Ferber M, Merryweather AT, Cayley PJ and Possee RD: Construction of an improved baculovirus insecticide containing an insect-specific toxin gene. Nature 352: 85-88, 1991.

20. Jennifer SC, Mark LH, Trevor W, Rosemary SH, David G, Bernadette MG, Timothy MC, Robert DP, Cayley PJ and Bishop DHL: Field trial of a genetically improved baculovirus insecticide. Nature 370: 138-140, 1994.

21. Matsuura Y, Possee RD, Overton HA and Bishop DHL: Baculovirus expression vectors: the requirements for high level expression of proteins, including glycoproteins. J Gen Virol 68 : 1233-1250, 1987

22. Luckow VA and Summers MD: Trends in the development of baculovirus expression vectors. Biotechnology 6: 47-55, 1988.

23. Hofmann C, Sandig V, Gennings G, Rudolph M, Schlag P and Strauss M: Efficient gene transfer into human hepatocytes by baculovirus vectors. Proc Natl Acad Sci USA 92: 10099-10103, 1995.

24. Boyce FM and Bucher NLR: Baculovirus-mediated gene transfer into mammalian cells. Proc Natl Acad Sci USA 93: 2348-2352, 1996.

25. Sandig V and Strauss M: Liver-directed gene transfer and application to therapy. J Mol Med 74: 205-212, 1996.

26. Pieroni L and La Monica N: Towards the use of baculovirus as a gene therapy vector. Curr Opin Mol Ther 3: 464-467, 2001.

27. Kost TA and Condreay JP: Recombinant baculoviruses as mammalian gene-delivery vectors. Trends Biotechnol 20 $173-180,2002$
28. Gronowski AM, Hilbert DM, Sheehan KCF, Garotta G and Schreiber RD: Baculovirus stimulates antiviral effects in mammalian cells. J Virol 73: 9944-9951, 1999.

29. Beck NB, Sidhu JS and Omiecinski CJ: Baculovirus vectors repress phenobarbital-mediated gene induction and stimulate cytokine expression in primary cultures of rat hepatocytes. Gene Ther 7: 1274-1283, 2000.

30. Kitajima M, Abe T,Miyano-Kurosaki N, Taniguchi M, Nakayama $\mathrm{T}$ and Takaku H: Induction of natural killer cell-dependent antitumor immunity by the Autographa californica multiple nuclear polyhedrosis virus. Mol Ther 16: 261-268, 2008.

31. Suzuki T, Chang MO, Kitajima M and Takaku H: Baculovirus activates murine dendritic cells and induces non-specific NK cell and T cell immune responses. Cell Immunol 262: 35-43, 2010.

32. Suzuki T, Chang MO, Kitajima M and Takaku H: Induction of antitumor immunity against mouse carcinoma by baculovirusinfected dendritic cells. Cell Mol Immunol 7: 440-446, 2010.

33. Schöttker B and Schmidt-Wolf IGH: Pulsing with blast cell lysate or blast-derived total RNA reverses the dendritic cell-mediated cytotoxic activity of cytokine-induced killer cells against allogeneic acute myelogenous leukemia cells. Ger Med Sci doi: 10. 3205/000141, URN: urn:nbn:de:0183-0001410, 2011.

34. Reis e Sousa C: Dendritic cells in a mature age. Nat Rev Immunol 6: 476-483, 2006.

35. Pasare $C$ and Medzhitov R: Toll pathway-dependent blockade of $\mathrm{CD} 4{ }^{+} \mathrm{CD} 25^{+} \mathrm{T}$ cell-mediated suppression by dendritic cells Science 299: 1033-1036, 2003.

36. Shevach EM: Regulatory T cells in autoimmmunity*. Annu Rev Immunol 18: 423-449, 2000.

37. Maloy KJ and Powrie F: Regulatory T cells in the control of immune pathology. Nat Immunol 2: 816-822, 2001.

38. Piccirillo CA and Shevach EM: Cutting edge: control of $\mathrm{CD}^{+}$ $\mathrm{T}$ cell activation by $\mathrm{CD} 4{ }^{+} \mathrm{CD} 25^{+}$immunoregulatory cells. J Immunol 167: 1137-1140, 2001.

39. Sakaguchi S: Naturally arising Foxp3-expressing CD $25^{+} \mathrm{CD} 4^{+}$ regulatory $\mathrm{T}$ cells in immunological tolerance to self and non-self. Nat Immunol 6: 345-352, 2005.

40. Turk MJ, Guevara-Patiño JA, Rizzuto GA, Engelhorn ME, Sakaguchi S and Houghton AN: Concomitant tumor immunity to a poorly immunogenic melanoma is prevented by regulatory T cells. J Exp Med 200: 771-782, 2004.

41. Yu P, Lee Y, Liu W, Krausz T, Chong A, Schreiber H and Fu YX: Intratumor depletion of $\mathrm{CD}^{+}$cells unmasks tumor immunogenicity leading to the rejection of late-stage tumors. J Exp Med 201: 779-791, 2005.

42. Tani H, Nishijima M, Ushijima H, Miyamura T and Matsuura Y: Characterization of cell-surface determinants important for baculovirus infection. Virology 279: 343-353, 2001.

43. Akira S, Takeda K and Kaisho T: Toll-like receptors: critical proteins linking innate and acquired immunity. Nat Immunol 2. 675-680, 2001. 